\title{
Post-Processing in Cardiovascular Computed Tomography: Performance of a Client Server Solution versus a Stand-Alone Solution
}

\section{Bildnachverarbeitung in der kardiovaskulären Computertomografie: Performance von Client-Server- versus Einzelplatzlösung}

Authors

Affiliations
C. Lücke' ${ }^{1}$, B. Foldyna ${ }^{1}$, C. Andres ${ }^{1}$, S. Boehmer-Lasthaus ${ }^{2}$, M. Grothoff' ${ }^{1}$, S. Nitzsche ${ }^{1}$, M. Gutberlet ${ }^{1}$, L. Lehmkuhl ${ }^{1}$

Diagnostic and Interventional Radiology, University Leipzig - Heart Centre, Leipzig

${ }^{2}$ Imaging \& Therapy Division, Siemens Healthcare Sector, Erlangen

Key words
cardiac
vascular
CT
post-processing software
performance

received $\quad 13.10 .2013$ accepted $\quad 21.5 .2014$

Bibliography

DOI http://dx.doi.org/

10.1055/s-0034-1366726

Published online: 14.8.2014

Fortschr Röntgenstr 2014; 186:

1111-1121 @ Georg Thieme

Verlag KG Stuttgart · New York . ISSN 1438-9029

\section{Correspondence \\ Dr. Christian Lücke \\ Abteilung für Diagnostische und Interventionelle Radiologie, \\ Universität Leipzig - \\ Herzzentrum \\ Strümpellstr. 39 \\ 04289 Leipzig \\ Germany \\ Tel.: ++49/341/8651702 \\ Fax: ++49/3 41/8651803 \\ cluecke@gmx.de}

\section{Abstract}

$\nabla$

Purpose: To compare the performance of server-based (CSS) versus stand-alone post-processing software (ES) for the evaluation of cardiovascular CT examinations (cvCT) and to determine the crucial steps.

Materials and Methods: Data of 40 patients (20 patients for coronary artery evaluation and 20 patients prior to transcatheter aortic valve implantation [TAVI]) were evaluated by 5 radiologists with CSS and ES. Data acquisition was performed using a dual-source 128-row CT unit (SOMATOM Definition Flash, Siemens, Erlangen, Germany) and a 64-row CT unit (Brilliance 64, Philips, Hamburg, Germany). The following workflow was evaluated: Data loading, aorta and coronary segmentation, curved multiplanar reconstruction (cMPR) and $3 \mathrm{D}$ volume rendering technique (3D-VRT), measuring of coronary artery stenosis and planimetry of the aortic annulus. The time requirement and subjective quality for the workflow were evaluated.

Results: The coronary arteries as well as the TAVI data could be evaluated significantly faster with CSS $(5.5 \pm 2.9 \mathrm{~min}$ and $8.2 \pm 4.0 \mathrm{~min}$, respectively) than with ES $(13.9 \pm 5.2 \mathrm{~min}$ and $15.2 \pm 10.9 \mathrm{~min}$, respectively, $\mathrm{p} \leq 0.01$ ). Segmentation of the aorta (CSS: $1.9 \pm 2.0 \mathrm{~min}$, ES: $3.7 \pm 3.3 \mathrm{~min})$, generating cMPR of coronaries (CSS: $0.5 \pm 0.2 \mathrm{~min}$, ES: $5.1 \pm 2.6 \mathrm{~min}$ ), aorta and iliac vessels (CSS: $0.5 \pm 0.4 \mathrm{~min}$ and $0.4 \pm 0.4 \mathrm{~min}$, respectively, ES: $1.6 \pm 0.7 \mathrm{~min}$ and $2.8 \pm 3 \mathrm{~min}$, respectively) could be performed significantly faster with CSS than with ES with higher quality of CMPR, measuring of coronary stenosis and 3D-VRT $(\mathrm{p}<0.05)$.

Conclusion: Evaluation of cvCT can be accomplished significantly faster and better with CSS than with ES. The segmentation re- mains the most time-consuming workflow step, so optimization of segmentation algorithms could improve performance even further.

Key points:

- With client-server-based (CSS) and standalone solutions (ES), cardiovascular CT datasets can be evaluated reliably.

- Evaluation of cardiovascular CT can be performed faster and better with CSS than with ES.

- In particular the generating of curved reconstructions is faster with CSS than with ES.

- Segmentation of data is a crucial step for semiautomatic software (CSS and ES).

Citation Format:

- Lücke C, Foldyna B, Andres C et al. Post-Processing in Cardiovascular Computed Tomography: Performance of a Client Server Solution versus a Stand-Alone Solution. Fortschr Röntgenstr 2014; 186: 1111-1121

\section{Zusammenfassung \\ $\nabla$}

Ziel: Die Performance einer Client-Server- (CSS) und einer Einzelplatz-Softwarelösung (ES) bezüglich der Auswertung von kardiovaskulären CTStudien (cvCT) zu vergleichen und zeitrelevante Arbeitsschritte zu bestimmen.

Material und Methoden: Die cvCT von insgesamt 40 Patienten (20 zur Koronarbeurteilung und 20 vor kathetergestütztem Aortenklappenersatz [TAVI]) wurden von 5 Untersuchern mit CSS und ES ausgewertet. Die Datenakquisition erfolgte an einem Dual-Source-128-Zeilen-CT (SOMATOM Definition Flash, Siemens, Erlangen, Deutschland) sowie an einem 64-Zeilen-CT (Brilliance 64, Philips, Best, Niederlande). Folgende Untersuchungsschritte wurden analysiert: Laden der Daten, Segmentierung von Aorta und Koronararterien, Anfertigung gekrümmter multiplanarer 
Reformatierungen (cMPR) und 3D-Rekonstruktionen (3D-VRT), Vermessung von Koronarstenosen und des Aortenklappenanulus. Die benötigte Zeit und subjektive Qualität der Arbeitsschritte wurde ermittelt.

Ergebnisse: Sowohl die Koronar- als auch die TAVI-Evaluation gelang mit CSS schneller (5,5 $\pm 2,9$ min bzw. 8,2 $\pm 4,0 \mathrm{~min})$ als mit ES (13,9 $\pm 5,2$ min bzw. 15,2 $\pm 10,9 \mathrm{~min}, \mathrm{p} \leq 0,01)$. Die Segmentierung der Aorta (CSS: 1,9 $\pm 2,0 \mathrm{~min}, \mathrm{ES}: 3,7 \pm 3,3 \mathrm{~min}$ ), die Erstellung der cMPR der Koronararterien (CSS: 0,5 $\pm 0,2 \mathrm{~min}$, ES: 5,1 $\pm 2,6 \mathrm{~min}$ ), der Aorta und der Beckengefäße (CSS: 0,5 $\pm 0,4$ min bzw. 0,4 $\pm 0,4 \mathrm{~min}$, ES: $1,6 \pm 0,7 \mathrm{~min}$ bzw. $2,8 \pm 3 \mathrm{~min}$ ) gelingt signifikant schneller mit CSS im Vergleich zur ES bei jeweils besserer Qualität der cMPR sowie der Vermessung und 3D-VRT der Koronararterien $(\mathrm{p}<0,05)$.

Schlussfolgerung: Die Auswertung von cvCT mit CSS benötigt weniger Zeit und führt zu qualitativ besseren Ergebnissen als mit ES. Die Segmentierung der Daten verbleibt als zeitrelevanter Arbeitsschritt, sodass eine Optimierung von Segmentierungsalgorithmen zu weiteren deutlichen Zeitersparnissen führen könnte.

\section{Introduction}

\section{$\nabla$}

The rapid technological development of multi-row computed tomography (CT) in the last two decades has resulted in an increase in CT examinations on the whole, the number of CT series acquired per patient, and the data volume per CT series as well as in a drastic reduction in the acquisition time of CT series to a few seconds. The speed of data volume generation in radiological departments doubles approximately every 2 years. Since the number of radiologists has not doubled, productivity in relation to the number of images viewed per time period and radiologist has increased significantly and will increase further if this trend continues. It is therefore not surprising that the scope of semiautomatic evaluation software has increased substantially to simplify and accelerate data analysis and to provide support for radiologists in the diagnosis process. The goal of semiautomatic evaluation software is to perform standardizable post-processing steps in a targeted manner without action on the part of the radiologist in advance or after initialization by the user.

The noninvasive visualization of coronary arteries via CT has become an established method. Moreover, CT is a reliable tool for the preoperative evaluation of patients prior to cardiac surgery, in particular for the planning of transcatheter aortic valve implantation (TAVI). TAVI requires very precise knowledge of the specific anatomy of the aortic root which $\mathrm{CT}$ provides with the greatest reliability among all imaging modalities.

Since the visualization of both the coronary arteries and the aortic root requires high spatial and temporal resolution, cardiovascular CT also produces large amounts of data. Single-phase coronary CT datasets are representative of the data volume compared to other CT datasets and place high demands on segmentation algorithms. TAVI datasets are challenging for post-processing software in relation to segmentation algorithms as well as data volume. Differences in performance should therefore be most apparent with respect to these examinations.
The goal of the present study was to compare the performance of client-server-based post-processing software with conventional stand-alone software with respect to the evaluation of ECG-triggered cardiovascular CT examinations using coronary datasets and datasets prior to TAVI as an example.

\section{Materials and Methods \\ $\nabla$}

\section{Study design and patient data}

ECG-triggered CT datasets for a total of 40 patients were included with the clinical indications coronary evaluation $(n=20)$ and TAVI evaluation $(n=20)$. The datasets were randomly selected by one of the examiners from an examination pool including approximately 4000 examinations from January 2008 to March 2012. Written informed consent for the evaluation of the anonymized data was obtained from each patient. The average age of the coronary evaluation patients was $54 \pm 18$ years with the majority of the patients being male ( 14 men, 6 women). The average age of the preoperative TAVI imaging patients was $82 \pm 6$ years with the majority of the patients being female ( 6 men, 14 women).

\section{CT protocol}

The image data were acquired using a second-generation dual-source CT scanner with 128 rows (=DSCT; SOMATOM Definition Flash, Siemens, Erlangen, Germany) and a 64row CT scanner (=CT64; Brilliance 64, Philips, Best, Netherlands).

The coronary datasets were acquired with a DSCT scanner in 13 cases and via a CT64 scanner in 7 cases. On average, $84.5 \mathrm{ml}( \pm 20 \mathrm{ml})$ of contrast agent from various manufacturers with different iodine concentrations were used $(6 \mathrm{x}$ Ultravist 370, Iopromide $370 \mathrm{mgI} / \mathrm{ml}$, Bayer-Schering-Pharma AG, Berlin, Germany and $14 \times$ Imeron 400, Iomeprol $400 \mathrm{mg} / \mathrm{ml}$, Bracco, Milan, Italy). A prospectively triggered CT scan was performed using the high-pitch technique in 10 cases. Retrospective ECG triggering was performed in 10 cases. Older examinations were primarily acquired using the CT64 scanner and more recent examinations with the DSCT scanner.

For TAVI imaging the DSCT scanner was used in 10 cases and the CT64 scanner in 10 cases. Patients received an average dose of $93 \mathrm{ml}( \pm 15 \mathrm{ml})$ of contrast agent ( $10 \times$ Iopromide $370 \mathrm{mgI} / \mathrm{ml}$ and $10 \times$ Iomeprol $400 \mathrm{mg} / \mathrm{ml}$ ). The examinations on the DSCT scanner were performed in the high-pitch mode. In the case of the CT64 scanner, the heart was acquired using a retrospectively ECG-synchronized technique followed by immediate acquisition of the aorta ( $\bullet$ Table 1 ).

\section{System specifications}

The image data were post-processed with client-server software (CSS, syngo.via CT Cardio-Vascular Engine, Version VA10B, Siemens, Erlangen) with corresponding hardware and network architecture and with stand-alone software (ES, syngo MultiModality Workplace, Version VE50A, Siemens, Erlangen).

The CSS server has two Intel Xeon CPUs X5570 @ $2.93 \mathrm{GHz}$, 86 GB RAM, hard disk $4.5 \mathrm{~TB}, 4 \times 1$ gigabit network cards with Microsoft Windows Server 2008 R2 Enterprise x 64 version, Service Pack 1 (Build 7601) and was put into operation on $12 / 23 / 2010$. 
Table 1 Examination protocols for coronary evaluation and TAVI evaluation on the basis of the two CT scans.

\begin{tabular}{|c|c|c|c|c|c|c|}
\hline \multirow[b]{2}{*}{ parameter } & \multicolumn{3}{|c|}{ CT coronary angiography } & \multicolumn{3}{|c|}{ preoperative TAVI planning CT } \\
\hline & brilliance CT 64 & SOMATOM DSCT & & brilliance CT 64 & & SOMATOM \\
\hline protocol & helical & helical & $\begin{array}{l}\text { helical high } \\
\text { pitch }\end{array}$ & helical (heart) & helical (aorta) & $\begin{array}{l}\text { helical high } \\
\text { pitch }\end{array}$ \\
\hline ECG synchronization & retrospective & retrospective & prospective & retrospective & non-triggered & prospective \\
\hline triggering & bolus triggering & bolus triggering & bolus triggering & bolus triggering & min. delay & bolus triggering \\
\hline tube voltage (kV) & 120 & 100 & $100-120$ & 120 & 120 & 100 \\
\hline tube current intensity & $\begin{array}{l}800 \mathrm{mAs} / \text { layer } \\
\text { (eff: } 381 \mathrm{~mA} \text { ) }\end{array}$ & 320 ref. mAs & 370 ref. mAs & $\begin{array}{l}500 \mathrm{mAs} / \text { layer } \\
\text { (eff: } 238 \mathrm{~mA} \text { ) }\end{array}$ & $\begin{array}{l}200 \mathrm{mAs} / \text { layer } \\
\text { (eff: } 313 \mathrm{~mA} \text { ) }\end{array}$ & 320 ref. mAs \\
\hline pulsing & yes, $20 \%$ & yes, $20-4 \%$ & - & yes, $20 \%$ & - & - \\
\hline slice thickness (mm) & 0.67 & 0.6 & 0.6 & 1 & 0.8 & 0.6 \\
\hline increment (mm) & 0.33 & 0.4 & 0.4 & 0.5 & 0.8 & 0.3 \\
\hline pitch & 0.15 & 0.28 & 3 & 0.2 & 0.641 & 3 \\
\hline scan direction & caudo-cranial & caudo-cranial & cranio-caudal & cranio-caudal & cranio-caudal & cranio-caudal \\
\hline
\end{tabular}

The CSS clients were Intel Core2 CPUs 6600 @2.4 GHz with NVIDIA Quadro 2000 graphics card, 66.5 GB hard disk, 3GB RAM and 1 gigabit network card with Windows XP Professional 2002, Service Pack 3 as the operating system.

The segmented, switched network has a transmission rate of $1 \mathrm{Gbit}$. A core router is used to switch between the individual IP segments.

The ES involved a computer with an Intel Xeon processor, X5570 @ 2.93 GHz with 11.9 GB RAM, an NVIDIA Quadro FX 5800 graphics card and a total hard disk capacity of 741 GB and a 1 gigabit network card with Microsoft Windows XP Professional 64x version 2003 Service Pack 2 as the operating system and was put into operation on 2/18/2010.

\section{Post-processing analysis}

The datasets were randomly assigned to 5 examiners with different levels of CT experience $(1,2,5,10,15$ years). The individual work steps were explained to all examiners prior to the start of the study and each examiner performed 3 test runs for coronary and TAVI evaluation with CSS and ES. The image data sent to the server of the CSS were automatically subjected to preparatory segmentation that was completed when the data were loaded by the user. Every case was evaluated by the same person first with CSS and subsequently after at least 30 days with ES to minimize memory effects. The workflows "CT chest pain", "CT vascular", and "CT-TAVI planning" were used for CSS.ES evaluation was performed using the workflows "In-space" and "Circulation".

The following post-processing steps were defined:

- Coronary evaluation: Load the image data, vessel segmentation (place a vascular path in the right coronary artery (RCA) and left coronary artery (LCA), including the branches: ramus interventricularis anterior (LAD) and ramus circumflexus (LCX)) ( $\bullet$ Fig. 1a, b), generate curved multiplanar reconstructions (cMPRs) of the abovementioned coronary arteries, measure coronary stenosis, if present, acquire 3D-VRT scan, generate end-diastolic short-axis images with a slice thickness of $5 \mathrm{~mm}$ and an increment of $5 \mathrm{~mm}$ (SA-MPR).

- TAVI evaluation: Load the image data, segment the aorta, generate a cMPR of the aorta, segment the peripheral arteries (PA): common iliac artery, common external artery, and common femoral artery on each side, generate
CMPRs of the PA, measure pelvic artery stenosis, if present ( $\bullet$ Fig. 2a, b), otherwise measure the PA, determine the distances between the coronary artery ostia and the valve plane, determine the effective diameter of the aortic annulus ( $\bullet$ Fig. 3a, b).

A maximum time of 15 minutes was set for a work step that could not be performed immediately after the preceding step.

The subjective quality of the results of the particular work steps was evaluated on a scale of $1-4$ (1=very good, 2 =good, 3 =diagnostically sufficient, 4 = unable to be evaluated). The quality of the cMPRs of the coronary arteries, the stenosis measurement, the 3D-VRT scans, and the SAMPRs was defined as a target variable in the case of the coronary evaluation. The quality of the cMPRs of the aorta, cMPRs of the PA stenosis measurement or diameter measurement of the PA, distance measurement between the coronary ostia and the valve plane and measurement of the aortic valve annulus was assessed for the TAVI evaluation.

\section{Statistical analysis}

Statistical evaluation was performed using the software IBM SPSS Statistics 20 (IBM Deutschland, Ehningen, Germany). The duration of the individual work steps was checked for significance using a one-way ANOVA, and the subjective quality was checked using the Pearson's chi-square test. The significance level was defined as $\mathrm{p}<0.05$.

\section{Results}

The cases were distributed according to CT experience as follows (CT experience, coronary evaluation, TAVI evaluation): 1 year: 4 and 2, 2 years: 3 and 5, 5 years: 9 and 4, 10 years: 3 and 8,15 years: 1 and 1 ).

\section{Coronary evaluation}

The total evaluation time as the sum of all evaluation steps was significantly shorter with CSS than with ES: $5.5 \pm$ $2.9 \mathrm{~min}$. or $332 \pm 174 \mathrm{~s}$ versus $13.9 \pm 5.2 \mathrm{~min}$. or $833 \pm 312 \mathrm{~s}$, $\mathrm{p}<0.01$ ( $\bullet$ Fig.4). Each individual work step also required less time with CSS than with ES in a pair-wise comparison (॰ Fig. 5, $\odot$ Table 2 ). 

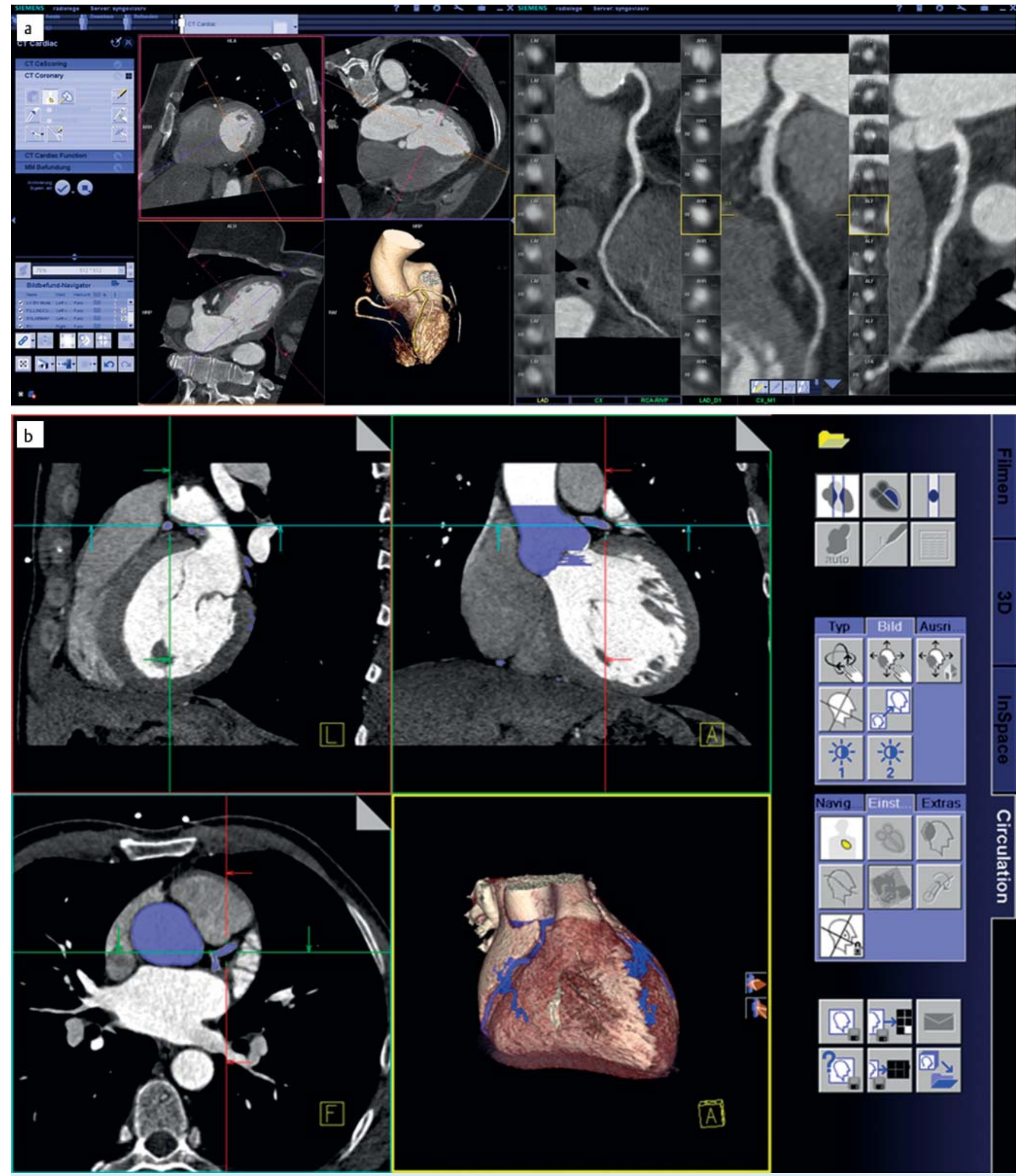

Fig. 1 a CSS segmentation of coronaries. On the left side MPRs in 2chamber view, 4-chamber view and short axis orientation as well as a 3DVRT are displayed. On the right side one can see CMPR of the 3 main coronary vessels (From left to right: Right coronary artery with posterior interventricular branch, left coronary artery with left descending artery (LAD) and circumflex branch (CX). b ES segmentation of coronaries. The segmentation of coronary arteries is performed semiautomatically. Coronary arteries are detected automatically and colored in blue or can be added manually with a plausibility check being performed beforehand by an algorithm.
The image data were loaded in the software significantly faster with CSS $(0.5 \pm 0.2 \mathrm{~min}$. $(28 \pm 10 \mathrm{~s}))$ than with ES $(0.4 \pm 0.1 \mathrm{~min}$. $(21 \mathrm{~s} \pm 8 \mathrm{~s})), \mathrm{p}=0.015$.
The segmentation of the coronary arteries comprised a large portion of the total evaluation time both for CSS and ES ( $45 \%$ vs. $28 \%$ ). At $2.5 \pm 2.3 \mathrm{~min}$. ( $151 \pm 139 \mathrm{~s})$ vs. $3.9 \pm$ $2.6 \mathrm{~min}$. (234 $\pm 155 \mathrm{~s})$, this was achieved slightly but not sig- 

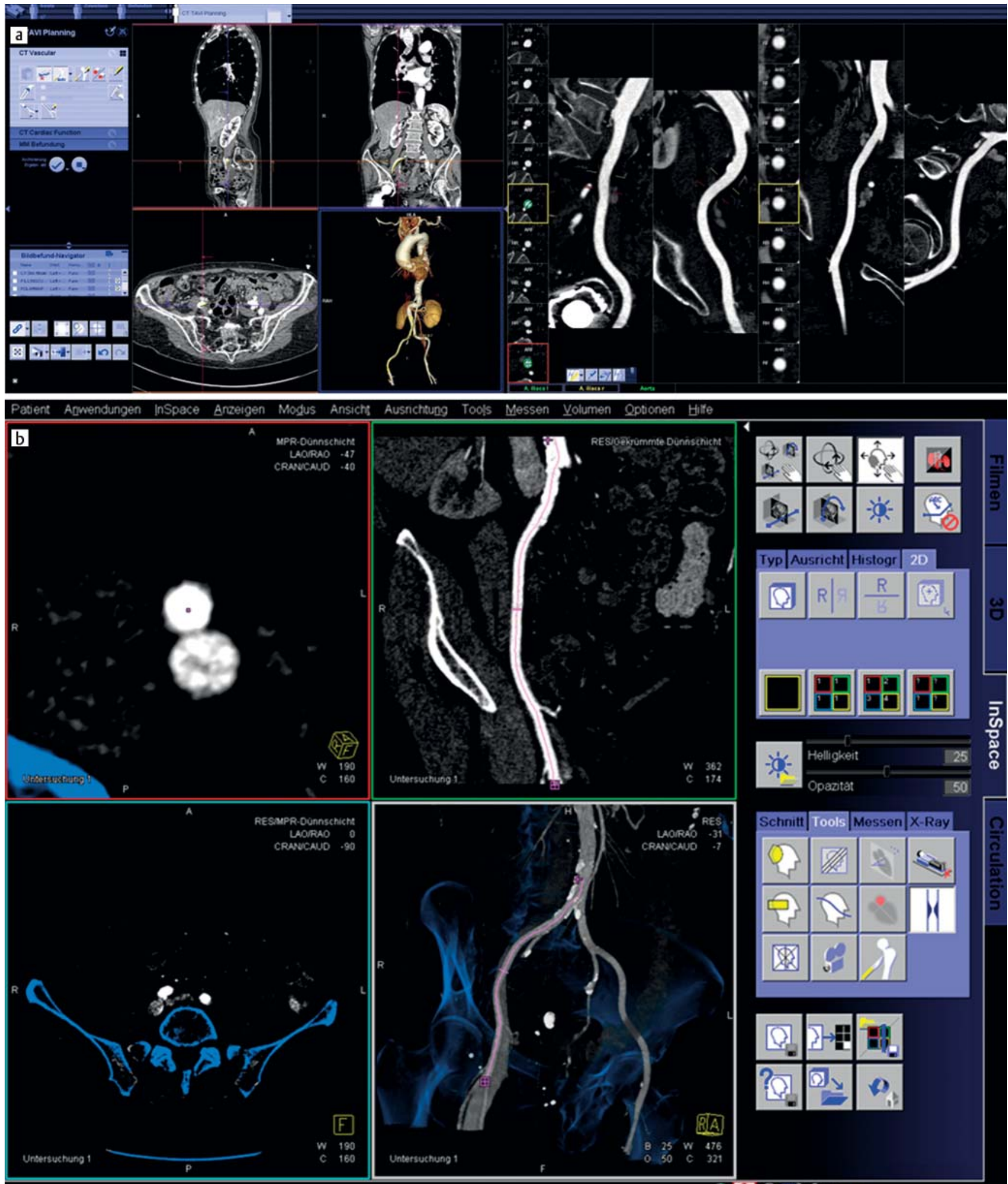

\section{(द) (ब)}

Fig. 2 a CSS TAVI evaluation. Evaluation of TAVI data sets is presented with standard MPRs in transverse, coronal and sagittal orientation, as well as a 3D-VRT. On the right panel cMPRs of the iliac vessels are depicted with the possibility to assess the diameters. Cross sections of the vessels are also displayed. b ES evaluation of iliac vessels. A cross section (upper left), a cMPR (upper right), the corresponding original transverse image of the iliac vessel and a 3D-VRT is displayed. nificantly faster with CSS than with ES ( $p=0.08)$. In 2 CSS cases and in 6 ES cases, the segmentation was incomplete. However, the difference was not significant $(p=0.12)$.
The faster generation of cMPRs and 3D-VRT scans which required only $20 \%$ of the total evaluation time with CSS in contrast to $53 \%$ of the total time with ES was largely respon- 


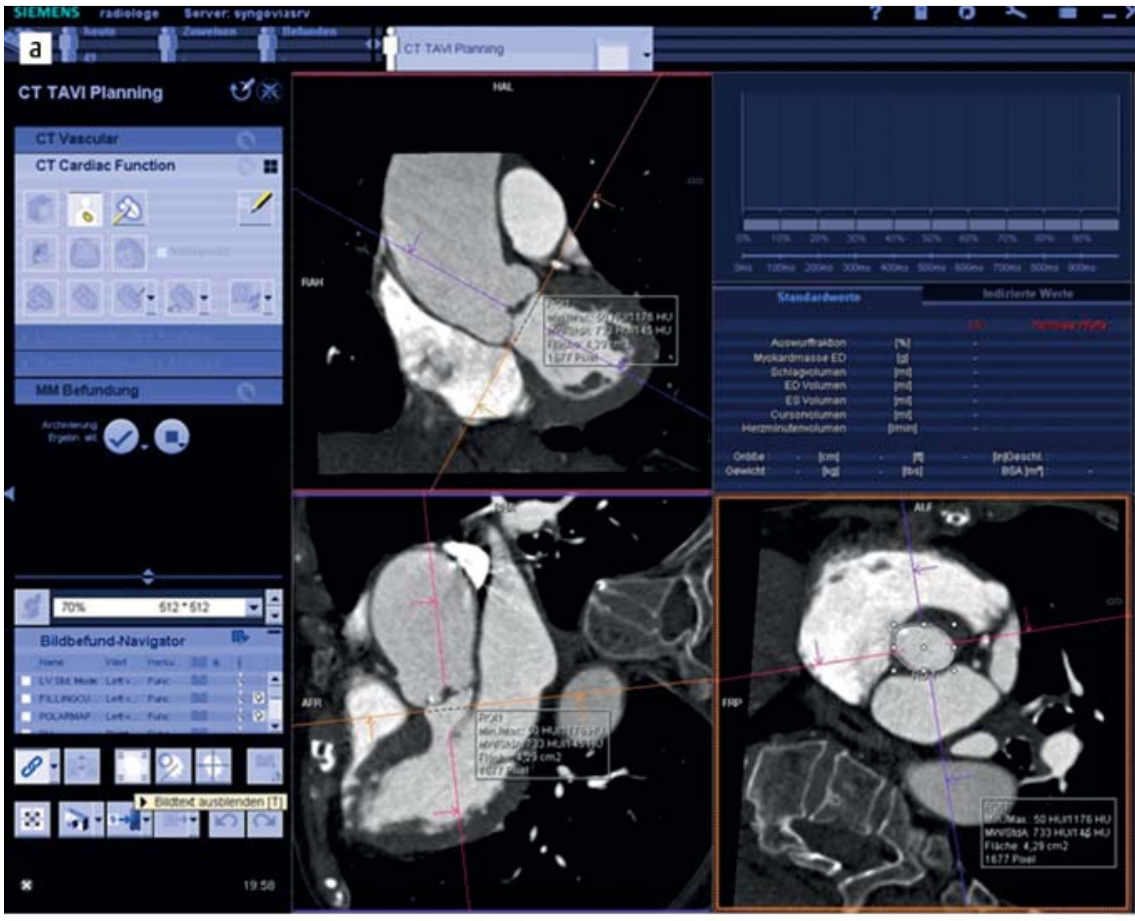

Fig. 3 a CSS measuring of aortic annulus. With the CSS three freely configurable image planes are displayed. The area of the aortic annulus can be measured and the effective diameter can be calculated. If a retrospectively gated data set was used, left ventricular volumes are visualized in the upper right window. In that case a high-pitch data set was used. b ES measuring of aortic annulus. The area of the aotic annulus can be measured in three orthogonal imaging planes, by which the effective diameter can be calculated. A 3D-VRT of the examination volume is depicted on the lower right.

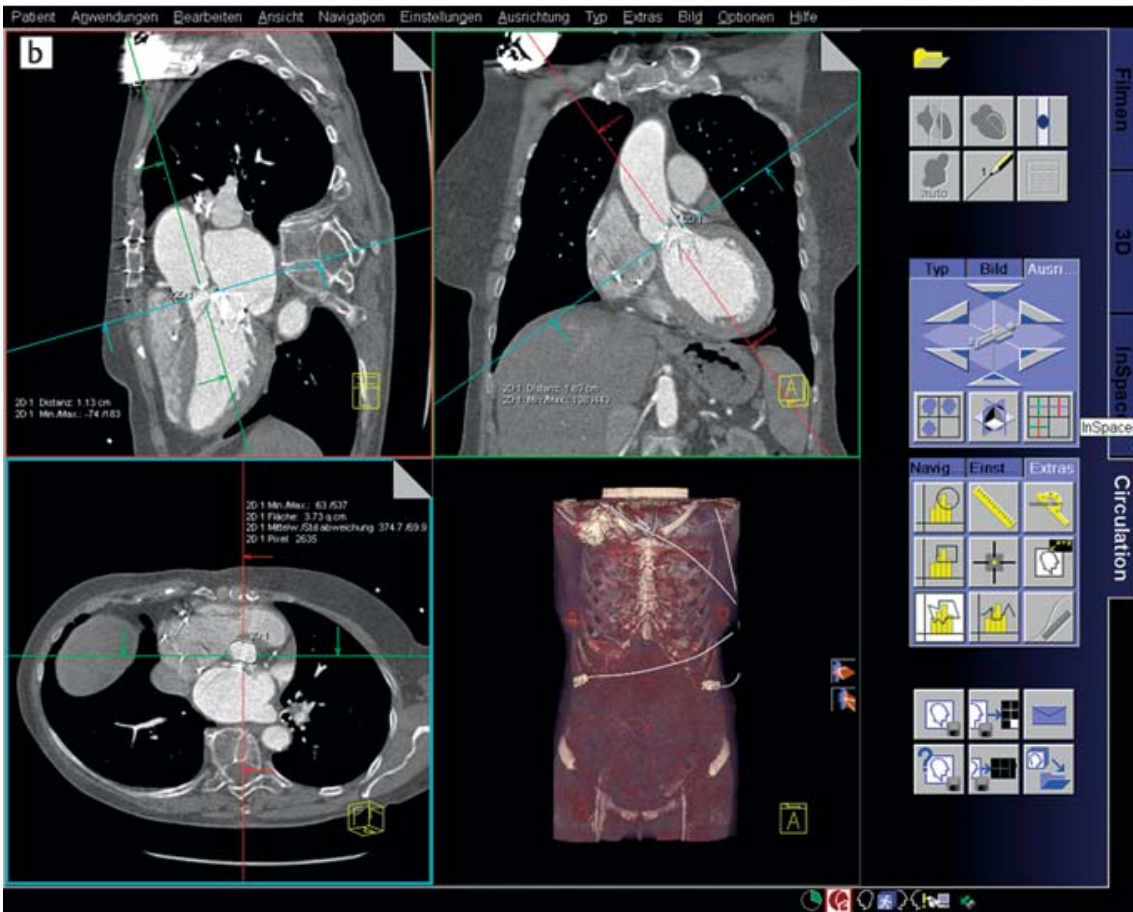

sible for the time difference in the total evaluation between CSS and ES. The cMPRs were generated 10 times faster with CSS $(0.5 \pm 0.2 \mathrm{~min}$. or $31 \pm 10 \mathrm{~s}$ vs. $5.1 \pm 2.6 \mathrm{~min}$. or $303 \pm$ $154 \mathrm{~s})$. The 3D-VRT scans were generated 4 times faster with CSS $(0.6 \pm 0.2 \mathrm{~min}$. $(37 \pm 12 \mathrm{~s}))$ than with ES $(2.3 \pm$ $1.5 \mathrm{~min}$. $(138 \pm 90 \mathrm{~s}))$. The quality of the cMPRs of the coronary arteries, the coronary stenosis measurement, the SAMPRs, and the 3DVRT scans was evaluated as sufficient or better in all cases ( $\bullet$ Fig. 6 ).

On average, the quality of the cMPRs of the coronary arteries with CSS was evaluated as "very good" (very good: 13, good: 5, sufficient: 2 ) and was significantly better compared to the cMPRs with ES (very good: 2, good: 6, sufficient: 12 ) which were evaluated on average as "sufficient" $(p<0.01)$. The quality of the 3D-VRT scans with CSS was also significantly better $(p<0.05)$. On average, it was evaluated as "very good" (very good: 13 , good: 5 , sufficient: 2 , while the quality with ES was evaluated only as "good" (very good: 2, good: 12 , sufficient: 6 ).

The stenosis measurement of the coronary arteries with CSS was significantly faster $(0.5 \pm 0.5 \mathrm{~min}$. $(32 \pm 28 \mathrm{~s})$ than with ES $(1.0 \pm 0.8 \mathrm{~min}$. $(57 \pm 48 \mathrm{~s})), \mathrm{p}=0.045$. The quality of the stenosis measurement via CSS was evaluated as "very good" (very good: 14, good: 5, sufficient: 1 ), while the quality with ES was evaluated on average as "good" (very good: 1, good: 17, sufficient: 2). 
An SA-MPR was generated with both modalities with only a slight time advantage for CSS $(1.0 \pm 0.4 \mathrm{~min}$. or $62 \pm 23 \mathrm{~s}$ vs. $1.2 \pm 0.9 \mathrm{~min}$. or $74 \pm 54 \mathrm{~s} ; \mathrm{p}=0.37$ ) and the quality for both modalities was evaluated on average as "very good" (CSS: very good: 17 , good: 3 , sufficient: 0 vs. ES: very good: 14 , good: 5 , sufficient: 1 ), $\mathrm{p}=0.41$.

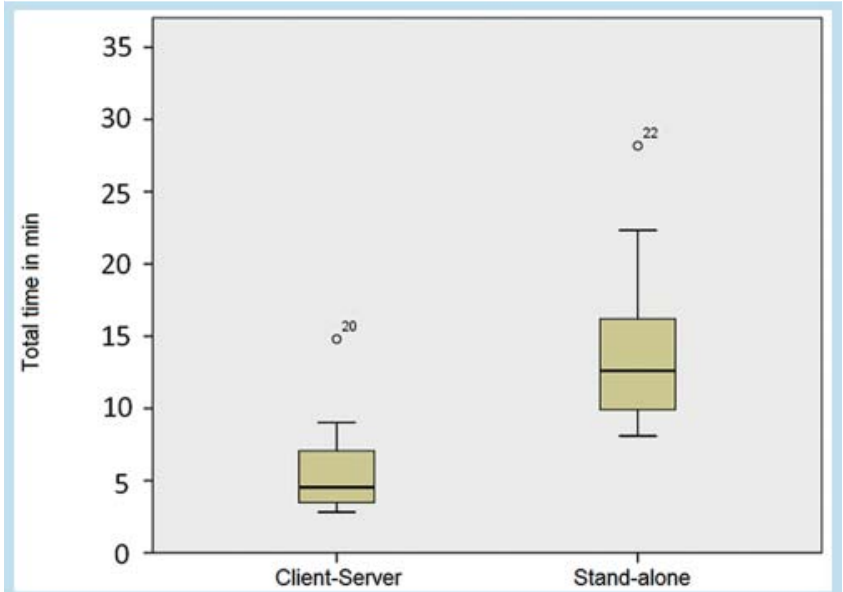

Fig. 4 Total time of coronary evaluation. Boxplot of the total time that was needed for the coronary evaluation. The median was $4.5 \mathrm{~min}$ with CSS and 12.6 min with ES.

\section{TAVI evaluation}

At $8.2 \pm 4$ min. $(490 \pm 240 \mathrm{~s})$, the total evaluation with CSS was on average almost twice as fast as the evaluation with ES $(15.2 \pm 10.9$ min. or $911 \pm 654 \mathrm{~s}, \mathrm{p}=0.01$; $\bullet$ Fig. 7$)$.

On average it took significantly longer to load the image data in the software with CSS $(97 \mathrm{~s}( \pm 97))$ than with ES (0.5 \pm 0.4 min. or $32 \pm 22$; $p<0.01$; $\bullet$ Fig. 8, $\diamond$ Table 3 ).

However, the segmentation of the aorta was on average significantly faster with CSS $(1.9 \pm 2 \mathrm{~min}$. or $114 \pm 121 \mathrm{~s})$ than with ES $(3.7 \pm 3.3 \mathrm{~min}$. or $224 \pm 200 \mathrm{~s} ; \mathrm{p}=0.04)$. At $0.5 \pm$ $0.8 \mathrm{~min}$. ( $29 \pm 50 \mathrm{~s})$, the segmentation of the PA was on average $4 \mathrm{x}$ faster with CSS than with ES $(2.0 \pm 3.2 \mathrm{~min}$. or 121 $\pm 194 \mathrm{~s} ; \mathrm{p}<0.05$ ). The complete segmentation of the aorta and the PA could be performed with CSS in all cases. Segmentation was incomplete in 3 cases with ES (15\%). In one case, the segmentation of the PA with ES was not possible so that the stenosis of the PA could not be evaluated.

The generation of cMPRs was 3x faster for the aorta (CSS: $0.5 \pm 0.5 \mathrm{~min}$. ( $32 \pm 27 \mathrm{~s}$ ) compared to ES: $1.6 \pm 0.7 \mathrm{~min}$. (98 \pm $42 \mathrm{~s}$ ), and almost $8 \mathrm{x}$ faster for the PA (CSS: $0.4 \pm 0.4 \mathrm{~min}$. (25 $\pm 24 \mathrm{~s})$ vs. ES: $2.8 \pm 3.0 \mathrm{~min}$. $(166 \pm 182 \mathrm{~s})$, in each case $\mathrm{p}<0.01)$. The quality of the cMPRs generated with CSS of the aorta (very good: 17 , good: 3 , sufficient: 0 ) as well as of the PA (very good: 17 , good: 3 , sufficient: 0 ) and was significantly better compared to the cMPRs generated with ES (aorta: very good: 11 , good: 4 , sufficient: 5 , p-0.04, PA: very good: 8 , good: 7 , sufficient: 4 , not able to be evaluated: $1, \mathrm{p}=0.02)(\diamond$ Fig. 6$)$.

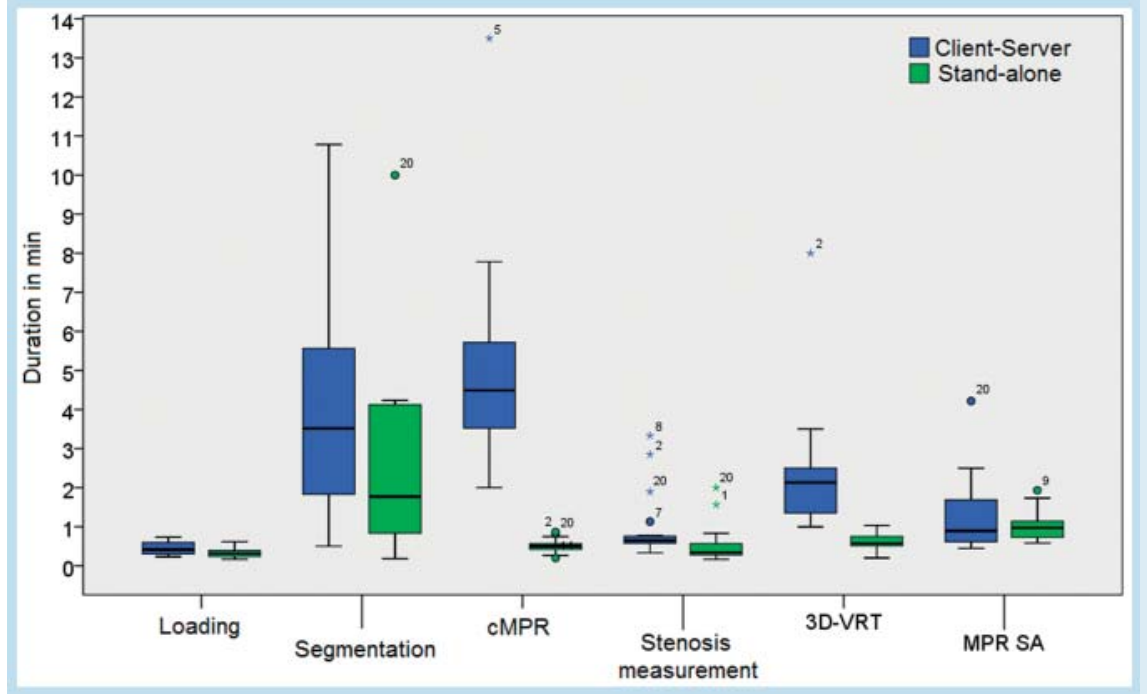

Fig. 5 Workflow steps of coronary evaluation. Duration of the different steps during coronary evaluation. The segmentation took 3.5 min with ES and 1.7 min with CSS. With both methods the segmentation was the most time-consuming work step (ES $45 \%$ vs. CSS $28 \%$ of the total time). Generation of CMPR showed the most relevant difference (ES: median: $4.536 \%$ of total time, CSS $0.5 \mathrm{~min}, 11 \%$ of total time).

Table 2 Measurement time of the individual steps of the coronary evaluation in seconds.

\begin{tabular}{|c|c|c|c|c|c|}
\hline coronary evaluation & client server & & stand-alone & & \\
\hline work steps & mean value $( \pm S D)$ & median (min, max) & mean value $( \pm S D)$ & median (min, max) & difference $( \pm S D)$ \\
\hline load images & $20.7( \pm 7.7)$ & $19(10.37)$ & $27.7( \pm 9.7)$ & $25(14.44)$ & $7.05( \pm 13.5)$ \\
\hline segmentation & $150.7( \pm 138.9)$ & $106(11.600)$ & $233.6( \pm 155.8)$ & $211(30.647)$ & $82.95( \pm 178.3)$ \\
\hline cMPR & $30.6( \pm 10)$ & $30(12.52)$ & $302.8( \pm 153.6)$ & $269.5(120.810)$ & $272.2( \pm 153.5)$ \\
\hline stenosis measurement & $31.5( \pm 28.2)$ & $20.5(10.120)$ & $57.3( \pm 48.1)$ & $39(20.200)$ & $25.85( \pm 52.9)$ \\
\hline 3D-VRT & $36.7( \pm 12.5)$ & $34.5(12.62)$ & $137.85( \pm 89.8)$ & $128(60.480)$ & $101.15( \pm 88.7)$ \\
\hline SA-MPR & $61.7( \pm 23.1)$ & $58.5(35.116)$ & $73.55( \pm 54.1)$ & $53.5(27.253)$ & $11.85( \pm 56.5)$ \\
\hline sum & $331.8( \pm 173.7)$ & $272(169.9)$ & $832.8( \pm 312.5)$ & 756 (485.1691) & $501.05( \pm 303.5)$ \\
\hline
\end{tabular}




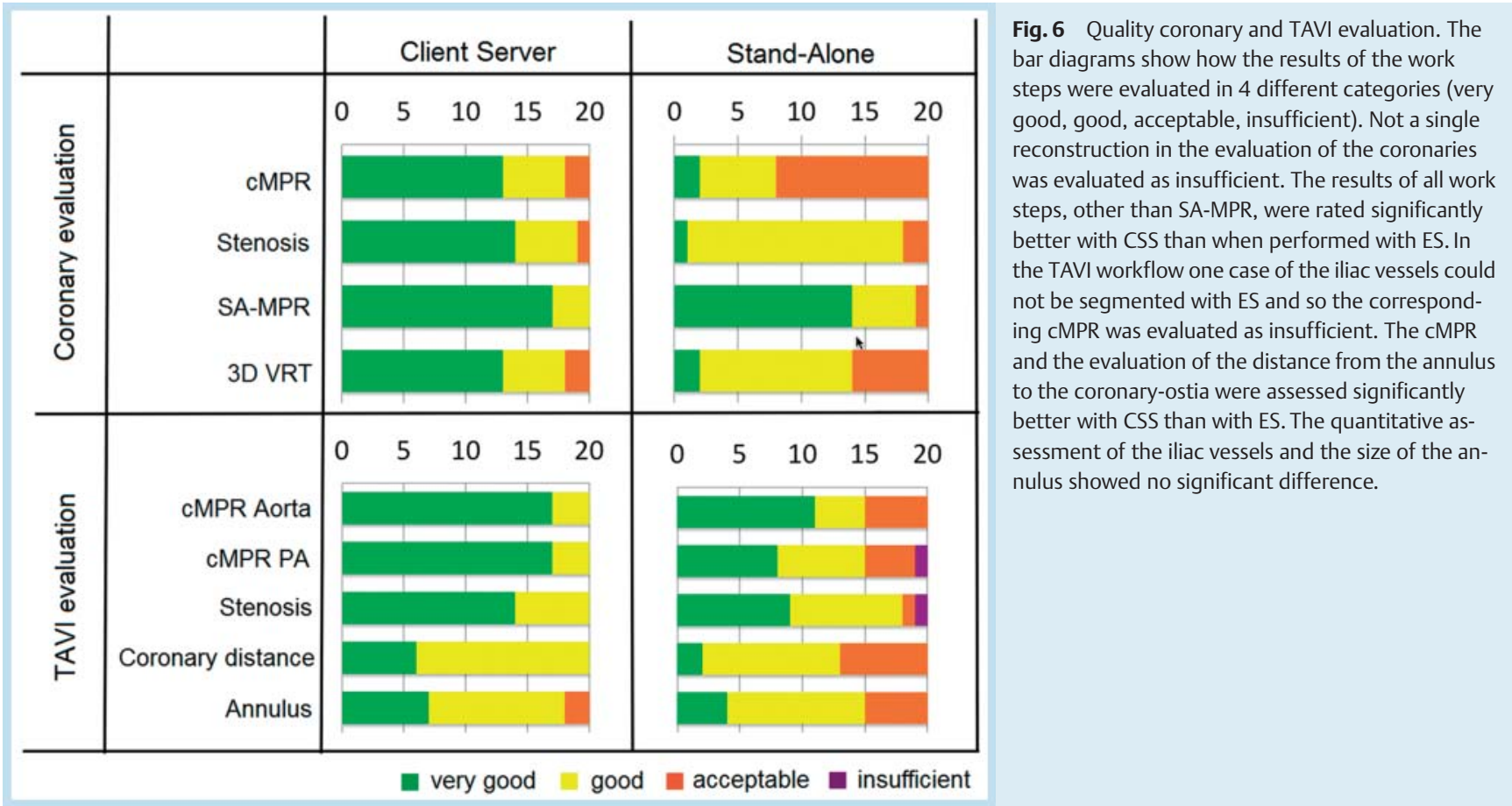

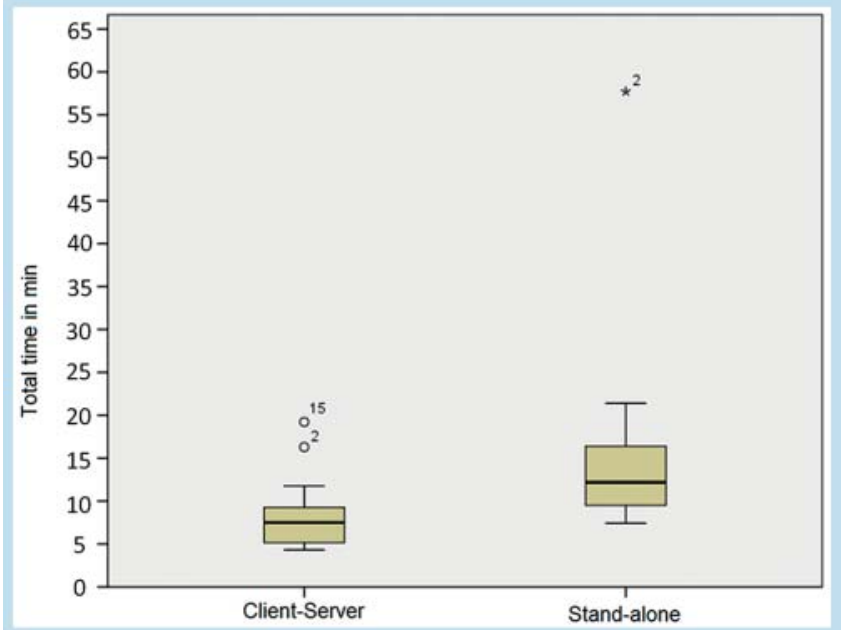

Fig. 7 Total time of TAVI evaluation. Boxplot of the total time needed for the TAVI evaluation. The median was 7.5 min with CSS and 12.2 min with ES

There were no significant differences in the stenosis measurement (CSS: $0.6 \pm 0.6 \mathrm{~min}$. (34 $\pm 34 \mathrm{~s}$ ), ES: $1.7 \pm 3.2 \mathrm{~min}$. $(100 \pm 193 \mathrm{~s}) ; \mathrm{p}=0.11)$, in the measurement of the distance of the coronary arteries from the valve plane (CSS: $1.8 \pm$ 0.9 min. (106 $\pm 52 \mathrm{~s}$ ), ES: $1.7 \pm 0.9 \mathrm{~min} .104 \pm 51 \mathrm{~s} ; \mathrm{p}=0.91$ ), and in the measurement of the aortic annulus (CSS: $0.9 \pm$ $0.7 \mathrm{~min}$. $(54 \pm 43 \mathrm{~s})$ vs. ES: $1.1 \pm 0.9 \mathrm{~min}$. $(67 \pm 56 \mathrm{~s}) ; \mathrm{p}=$ 0.39 ). The quality of the measurement of a stenosis or the diameter of the PA with CSS was not significantly better (very good: 14, good: 6, sufficient: 0 ) compared to the measurement with ES (very good: 9, good: 9, sufficient: 1, not able to be evaluated: $1, \mathrm{p}=0.3$ ). The presence of a stenosis did not significantly effect the vessel measurement time $(p=0.68)$ not even under consideration of the software en- vironment $(p=0.15)$. The quality of the determination of the distance of the coronary arteries from the valve plane with CSS was significantly better despite an identical median ("good") (very good: 6, good: 14, sufficient: 0) than the quality of the measurement with ES (very good: 2, good: 11, sufficient: 7). The quality of the aortic annulus measurement was not significantly different (CSS: very good: 7 , good: 11 , sufficient: 2 , ES: very good: 4 , good: 11 , sufficient: 5 ).

\section{Discussion \\ $\nabla$}

This paper analyzes the possible time efficiency of the semiautomatic evaluation of the coronary arteries and preoperative evaluation prior to TAVI.

Our study shows that both the evaluation of the coronary arteries and preoperative planning before TAVI can be reliably performed with both software environments. However, there are relevant and significant differences for both evaluation processes (coronary evaluation and TAVI evaluation). Semiautomatic evaluation with CSS is significantly faster than with ES and provides qualitatively better results. During coronary evaluation, segmentation of the coronary arteries comprises a significant portion of the total evaluation time (28\% (CSS) and $45 \%$ (ES)) and is significantly faster with CSS than with ES. During TAVI evaluation, segmentation of the aorta took $20 \%$ (CSS) and $21 \%$ (ES) of the total time with CSS still being significantly faster than ES.

The generation of cMPRs with CSS is performed partly in the background so that other work steps can be performed in the meantime. Queries regarding the generation of cMPRs, e.g. of the individual coronary arteries, can be sent in quick succession to the server with CSS so that the cMPRs are generated virtually in parallel. For the measurement of the generation of the cMPRs, the time from the query to the server to viewing of the cMPRs was measured for CSS even though 


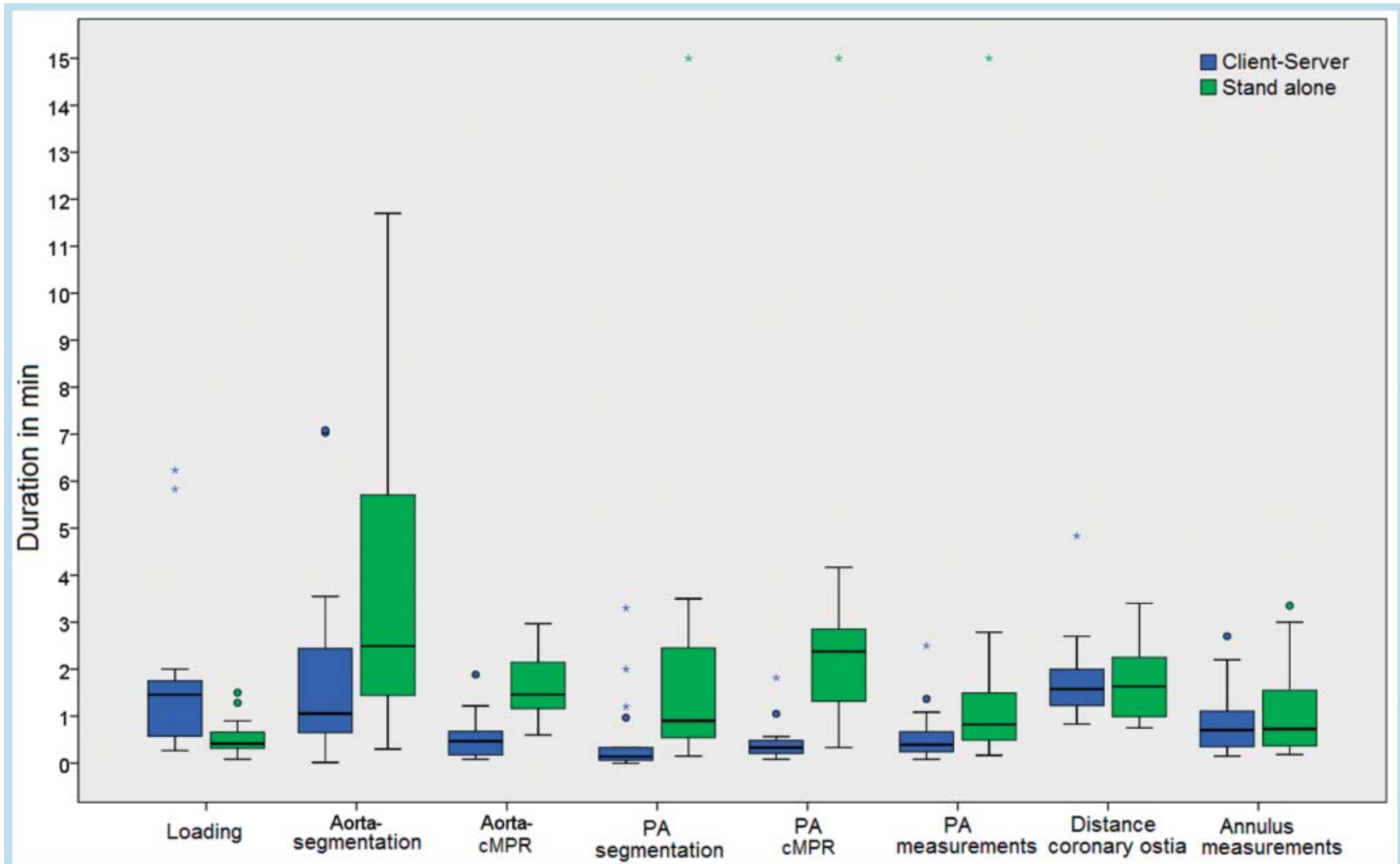

Fig. 8 Workflow steps of TAVI evaluation. Duration of the different work steps during TAVI evaluation. Segmentation was significantly faster with CSS than with ES, although based on the total time that was needed for the work step it took $21 \%$ with ES and $20 \%$ with CSS. Note that "loading of the

data" was faster with ES than with CSS. The quantitative assessment of the iliac vessels, the area of the aortic annulus as well as the distance of the coronaries to the aortic valve showed no significant difference.

Table 3 Measurement time of the individual steps of the TAVI evaluation in seconds.

\begin{tabular}{|lccccc|}
\hline TAVI evaluation & client server & & stand-alone & & \\
work steps & mean value $( \pm$ SD) & median $(\mathbf{m i n}, \mathbf{m a x})$ & mean value $( \pm$ SD) & median (min, max) & difference ( \pm SD) \\
\hline load images & $96.4( \pm 97.4)$ & $87.5(16.374)$ & $31.9( \pm 21.7)$ & $25(5.90)$ & $-64.5( \pm 100.8)$ \\
\hline aorta segmentation & $114.4( \pm 120.6)$ & $63.5(1.425)$ & $223.9( \pm 200.1)$ & $149.5(18.702)$ & $109.55( \pm 233.9)$ \\
\hline aorta cMPR & $32.1( \pm 26.8)$ & $28(5.113)$ & $97.9( \pm 42.4)$ & $87.5(36.178)$ & $65.75( \pm 37.7)$ \\
\hline BB segmentation & $29( \pm 49.9)$ & $8.5(0.198)$ & $121( \pm 194.4)$ & $54(9.900)$ & $92( \pm 165.2)$ \\
\hline BB cMPR & $25( \pm 23.7)$ & $20(5.109)$ & $165.8( \pm 182.1)$ & $142.5(20.900)$ & $140.8( \pm 186.9)$ \\
\hline stenosis measurement & $34.3( \pm 34)$ & $23.5(5.150)$ & $99.5( \pm 192.5)$ & $49.5(10.900)$ & $65.25( \pm 196.6)$ \\
\hline coronary distances & $105.6( \pm 52.4)$ & $94.5(50.290)$ & $103.7( \pm 50.7)$ & $98(45.204)$ & $-1.95( \pm 78)$ \\
\hline annulus & $53.5( \pm 43)$ & $42(9.162)$ & $67.3( \pm 56.3)$ & $43.5(11.201)$ & $13.8( \pm 70.1)$ \\
\hline total time & $490.2( \pm 239.9)$ & $450.5(259.1153)$ & $910.9( \pm 654.5)$ & $731.5(446.3463)$ & $420.7( \pm 606.7)$ \\
\hline
\end{tabular}

further work steps would have been possible. In the case of $\mathrm{ES}$, the calculation is performed on the workstation thus preventing further work steps. The generation of cMPRs with ES can thus only be performed in a serial manner. The generation of cMPRs as well as VRT scans is thus significantly faster with CSS, virtually at the push of a button, while multiple actions are necessary with ES to generate quality cMPRs and VRT scans.

The evaluation of the TAVI datasets could be performed with CSS with the "CT chest pain", "CT vascular", and "CT TAVI planning" workflows. While there was no significant difference between the evaluation workflows during the individual work steps, the "CT TAVI planning" workflow had a longer loading time. If the datasets were loaded with " $C T$ vascular" or "CT chest pain", the average loading time for CSS was $1.0 \pm 0.5 \mathrm{~min}$. $(60 \pm 32 \mathrm{~s})$. The difference with respect to ES was statistically significant $(\mathrm{p}<0.01)$. A possible reason for the longer loading time could be the automatic segmentation steps in the "CT TAVI planning" workflow. In the case of multi-phase datasets, all heart phases are segmented to determine functional parameters (e.g. ejection fraction).

The segmentation of the coronary arteries was incomplete in 2 cases with CSS and in 6 cases with ES. While a path can also be placed with CSS along a vessel that was previously not correctly segmented, the evaluation with ES requires 
prior correct segmentation that can only be performed semiautomatically and not purely manually and thus remains incomplete.

Segmentation was incomplete for TAVI evaluation in three cases with ES. In one case the evaluation of the PA with ES was not possible despite very good contrast of the PA with respect to the surrounding tissue and the evaluation via CSS was unproblematic and complete. The subsequently affected examination steps (generation of the cMPRs of the PA and stenosis measurement) were thus also not possible and the examination time was set to 15 minutes for these work steps. In the other two cases the segmentation with ES was incomplete but the subsequent generation of cMPRs and the stenosis measurement were possible without a relevant loss of information.

For the evaluation of brain-supplying vessels, Tsiflikas et al. as well as Gerhards et al. were able to show that semiautomatic evaluation results in a time savings and reproducible results. In relation to cardiovascular issues, it was able to be shown that semiautomatic segmentation software is useful for the evaluation of LV function and results in a time savings compared to manual evaluation. Coronary evaluation is partly faster via semiautomatic software and partly slower than the manual approach but always with the same or better quality and lower interobserver variability. Reimann et al. determined the segmentation work step as the relevant work step to be improved in order to accelerate evaluation. The time we needed for the segmentation of the coronary arteries with ES corresponds to the values determined by Reimann et al. $(3.9 \pm 2.6 \mathrm{~min}$. on average in our study vs. $4.2 \mathrm{~min}$ in the study by Reimann et al.) and was significantly faster with CSS (on average $2.5 \pm 2.3 \mathrm{~min}$.). The evaluation of PA in preinterventional planning in stent graft implantation has a lower interobserver variability using semiautomatic software compared to manual evaluation which highlights the validity of performing such measurements with semiautomatic software. As a result of further developments in the field of TAVI evaluation, there is now a solution that automatically displays and measures the aortic annulus. It is expected that this automation will allow a further time savings in the evaluation of the aortic annulus and the selection of the correct implant.

\section{Limitations}

We did not separately study the system-inherent difference in network architecture, processing power, and data transmission from the memory of the CT unit or a long-term memory to a server or a workstation since it is very dependent on the network architecture and different hospitals use different configurations so that measured values are not easily transferable. We indicated our network and hardware specifications accordingly. Identical features with respect to the processing power of the server and the workstation would have meant a disadvantage for the client server solution, incurred significant costs, and still would not have provided comparable results. The conditions were reproduced as realistically as possible, i.e., the evaluations were performed parallel to the clinical routine in both cases. As a result it cannot be ruled out that the server was simultaneously being used for tasks from other clients.

The parallel work of multiple radiologists on different examinations from different workstations was not taken into consideration in our study. That is an important factor in increasing the efficiency of hospital radiology. A stand-alone workstation currently represents a bottleneck in an active CT department.

A correlation between experience in CT and speed/quality of the evaluation was explicitly not performed to rule out a competitive effect among the examiners.

\section{Conclusion}

The use of CSS results in a time savings in the evaluation of coronary arteries and the preoperative evaluation prior to TAVI compared to ES. This time savings is necessary since radiologists are confronted with a continuously increasing number of images. This applies in particular for the evaluation of cardiovascular datasets since a high spatial as well as temporal resolution is needed. Cardiovascular CT datasets thus place higher demands on post-processing software and segmentation algorithms. CSS allows evaluation of coronary arteries as well as preoperative evaluation prior to TAVI faster and better than ES. The generation of cMPRs with ES and segmentation with both software solutions were identified as relevant time-intensive work steps. Future software development should therefore retain the advantage of fast cMPR generation and improve the algorithm for segmenting coronary arteries or vessels to maximize the time savings. The advantage that multiple radiologists can work at the same time on different workstations on different cases is not even taken into consideration. The examination of these factors in relation to increased efficiency of a hospital radiology department is a topic for future studies.

\section{Clinical relevance of the study}

- Semiautomatic evaluation software represents a possibility to simplify and accelerate data analysis and to support radiologists in the diagnosis process with cardiovascular CT datasets being able to be reliably evaluated both with a client-server-based software environment (CSS) and a stand-alone solution (ES).

- The use of client-server-based software (CSS) compared to the use of stand-alone software (ES) results in a time savings in the evaluation of cardiovascular CT datasets which is necessary since radiologists are facing a continuously increasing number of images.

- The evaluation of cardiovascular CT datasets is faster and better with CSS than with ES, with the up to $10 \mathrm{x}$ faster generation of curved multiplanar reconstructions and the faster segmentation algorithms being particularly responsible for the time savings. However, the segmentation of data remains a time-relevant work step so that optimization of segmentation algorithms could result in a further significant time savings.

\section{References}

1 Achenbach S, Marwan M, Schepis T et al. High-pitch spiral acquisition: a new scan mode for coronary CT angiography. Journal of cardiovascular computed tomography 2009; 3: 117-121

2 Boehm T, Handgraetinger 0 , Link $J$ et al. Evaluation of radiological workstations and web-browser-based image distribution clients for a 
PACS project in hands-on workshops. European radiology 2004; 14 : 908-914

3 Kotter E, Langer M. Computer aided detection and diagnosis in radiology. European radiology 2011; 21: 590-592

4 Tsiflikas I, Biermann C, Thomas C et al. Carotid artery stenosis: performance of advanced vessel analysis software in evaluating CTA. European journal of radiology 2012; 81: 2255-2259

5 Kopetsch T. Entwicklung der Altersstruktur und der Zahl der Ärzte in Deutschland unter besonderer Berücksichtigung der Radiologen. Deutscher Röntgenkongress 2010; 182: S66

6 Andres C, Gutberlet M, Lehmkuhl L. Preoperative CT imaging in infectious endocarditis before urgent indication for heart valve replacement: whole body computerized tomography including coronary evaluation. Fortschr Röntgenstr 2013; 185: 373-375

7 Achenbach S, Barkhausen J, Beer $M$ et al. Konsensusempfehlungen der DRG/DGK/DGPK zum Einsatz der Herzbildgebung mit Computertomografie und Magnetresonanztomografie. Fortschr Röntgenstr 2012; 184: $345-368$

8 Arbab-Zadeh A, Miller JM, Rochitte CE et al. Diagnostic accuracy of computed tomography coronary angiography according to pre-test probability of coronary artery disease and severity of coronary arterial calcification. The CORE-64 (Coronary Artery Evaluation Using 64-Row Multidetector Computed Tomography Angiography) International Multicenter Study. Journal of the American College of Cardiology 2012; 59: 379-387

9 Holmes DR Jr, Mack MJ, Kaul S et al. 2012 ACCF/AATS/SCAI/STS Expert Consensus Document on Transcatheter Aortic Valve Replacement: Developed in collaboration with the American Heart Association, American Society of Echocardiography, European Association for Cardio-Thoracic Surgery, Heart Failure Society of America, Mended Hearts, Society of Cardiovascular Anesthesiologists, Society of Cardiovascular Computed Tomography, and Society for Cardiovascular Magnetic Resonance. The Annals of thoracic surgery 2012; 93: 1340 - 1395

10 Lehmkuhl L, Foldyna B, Von Aspern K et al. Inter-individual variance and cardiac cycle dependency of aortic root dimensions and shape as assessed by ECG-gated multi-slice computed tomography in patients with severe aortic stenosis prior to transcatheter aortic valve implantation: is it crucial for correct sizing? The international journal of cardiovascular imaging 2013; 29: $693-703$
11 Kempfert J, Van Linden A, Lehmkuhl L et al. Aortic annulus sizing: echocardiographic versus computed tomography derived measurements in comparison with direct surgical sizing. European Journal of CardioThoracic Surgery 2012; 42: 627-633

12 Gerhards A, Raab P, Herber S et al. Software-assisted CT-postprocessing of the carotid arteries. Fortschr Röntgenstr 2004; 176: 870-874

13 Beyer F, Zierott L, Fallenberg EM et al. Comparison of sensitivity and reading time for the use of computer-aided detection (CAD) of pulmonary nodules at MDCT as concurrent or second reader. European radiology 2007; 17: $2941-2947$

14 Dewey $M$, Schnapauff $D$, Laule $M$ et al. Multislice CT coronary angiography: evaluation of an automatic vessel detection tool. Fortschr Röntgenstr 2004; 176: 478 - 483

15 Busch S, Johnson TR, Nikolaou K et al. Visual and automatic grading of coronary artery stenoses with 64-slice $\mathrm{CT}$ angiography in reference to invasive angiography. European radiology 2007; 17: 1445-1451

16 Cordeiro MA, Lardo AC, Brito MS et al. CT angiography in highly calcified arteries: 2D manual vs. modified automated 3D approach to identify coronary stenoses. The international journal of cardiovascular imaging 2006; 22: 507-516

17 Reimann AJ, Tsiflikas I, Brodoefel $\mathrm{H}$ et al. Efficacy of computer aided analysis in detection of significant coronary artery stenosis in cardiac using dual source computed tomography. The international journal of cardiovascular imaging 2009; 25: 195-203

18 Diehm N, Baumgartner I, Silvestro A et al. Automated software supported versus manual aorto-iliac diameter measurements in CT angiography of patients with abdominal aortic aneurysms: assessment of interand intraobserver variation. VASA Zeitschrift für Gefäßkrankheiten 2005; 34: $255-261$

19 Lutz AM, Willmann JK, Pfammatter T et al. Evaluation of aortoiliac aneurysm before endovascular repair: comparison of contrast-enhanced magnetic resonance angiography with multidetector row computed tomographic angiography with an automated analysis software tool. Journal of vascular surgery 2003; 37: 619-627

20 Blanke P, Spira EM, Ionasec $R$ et al. Semiautomated Quantification of Aortic Annulus Dimensions on Cardiac CT for TAVR. J Am Coll Cardiol Img 2014; 7: 320-322 